\title{
Accuracy of determination of the glomerular filtration marker iohexol by European laboratories as monitored by external quality assessment
}

https://doi.org/10.1515/cclm-2018-1175

Received November 1, 2018; accepted January 17, 2019; previously published online May 10, 2019

\section{Abstract}

Background: Glomerular filtration is the most important kidney function. The most accurate glomerular filtration rate (GFR) estimates are based on the clearance of exogenous filtration markers. Of these, iohexol is the only exogenous marker that is included in an external quality assessment (EQA) scheme. The aim of the present study was to evaluate the performance of the European laboratories participating in Equalis' EQA scheme for iohexol.

Methods: Weighed amounts of iohexol (Omnipaque) were added to plasma samples and distributed to laboratories participating in the EQA scheme for iohexol. All laboratories performed the assays in a blinded fashion.

Results: The number of participating laboratories varied between 27 and 34 during the study period. Iohexol was determined by HPLC in $77 \%$ of the laboratories and by UPLC/MS/MS methods in $15 \%$ of the laboratories. The mean interlaboratory coefficient of variation was $4.7 \%$ for the HPLC methods and $6.4 \%$ for the UPLC/MS/MS methods. The mean bias between calculated and measured iohexol values was $-1.3 \mathrm{mg} / \mathrm{L}$ (95\% confidence interval \pm 0.3 ) during the first part of the study period and $0.1 \mathrm{mg} / \mathrm{L}( \pm 0.3)$ during the later part.

Conclusions: The low interlaboratory variation demonstrates that iohexol can be measured reliably by

*Corresponding author: Anders Larsson, Department of Medical Sciences, Section of Clinical Chemistry, Uppsala University, Akademiska Sjukhuset, Entrance 61, 3rd Floor, Uppsala SE-75185, Sweden, Phone: +4618 61100 00, Fax: +4618 61137 03,

E-mail: anders.larsson@akademiska.se

Gunnar Nordin, Sara Ekvall and Carolina Kristoffersson: Equalis, Uppsala, Sweden

Ann-Sofie Jonsson: Department of Clinical Chemistry, Landstinget i Värmland, Karlstad, Sweden

Sten-Erik Bäck: Department of Clinical Chemistry, Lund University Hospital, Lund, Sweden

Niclas Rollborn: Department of Medical Sciences, Section of Clinical Chemistry, Uppsala University, Uppsala, Sweden many laboratories and supports the use of iohexol as a GFR marker when there is a need for high quality GFR measurements.

Keywords: external quality assessment scheme; GFR; glomerular filtration rate; iohexol.

\section{Introduction}

Glomerular filtration rate (GFR) measurements are essential for monitoring patients with suspected kidney diseases and for verifying normal kidney function in living kidney donors. The gold standard method for measuring GFR has been renal clearance of inulin measured during continuous infusion and urine collection [1]. This is a cumbersome method rarely used today, and inulin has been substituted with other exogenous filtration markers such as ${ }^{99 \mathrm{~m}} \mathrm{Tc}$-diethylenetriaminepentaacetic acid (99mTc-DTPA), chromium 51-labeled ethylenediaminetetraacetic acid $\left({ }^{51} \mathrm{Cr}\right.$-EDTA), iohexol and iothalamate [2]. Several of these markers are radiopharmaceuticals meaning they have short half-lives, expose the patient to radiation and are more expensive [3].

In contrast, iohexol is a non-ionic, non-radioactive X-ray contrast medium $[4,5]$, sold under brand names such as Omnipaque and Hexopaque [6]. Iohexol is also available as a density gradient medium under the names Nycodenz, Histodenz and Accudenz [7, 8]. For more than 30 years, iohexol has been used as an exogenous GFR marker. Furthermore, iohexol is considered as one of the safer exogenous GFR markers [9]. The amount of iohexol administered for GFR measurements is much smaller, typically $5 \mathrm{~mL}$ or less, than when used as a contrast medium. It is also sufficient to measure the plasma concentration of iohexol, eliminating cumbersome urine collections and simplifying the procedure.

Certified reference materials for iohexol or any other of the exogenous GFR markers are as yet unavailable. Instead, laboratories performing iohexol measurements use the registered contrast medium formulation for calibration. As this is highly concentrated, serial dilution in 
several steps is required to ensure relevant iohexol levels for calibration; a procedure that may introduce error. To verify that the assay is without bias, participation in external quality assessment (EQA) schemes is important. The only EQA scheme in Europe for iohexol GFR determination is managed by Equalis, a nonprofit EQA organization in Sweden. The aim of the present study was to evaluate the performance of the participating European laboratories in this EQA scheme.

\section{Materials and methods}

\section{EQA material}

The results of a total of 14 EQA rounds sent out from Equalis (Uppsala, Sweden) during the time period 2015-2018 were investigated. Each EQA round consisted of two samples. For preparation of the EQA materials, Omnipaque (300 mg I/mL, GE Healthcare Ireland, Cork, Ireland) was diluted (weight values) in pooled plasma from healthy blood donors to achieve calculated iohexol concentrations in the range of 20-105 mg/L. The absence of iohexol in the original plasma pool was verified by HPLC. The method was only used to verify that there was no iohexol in the samples prior to addition of the iohexol to the EQA samples. The values assigned to the EQA samples were either the consensus value reported by the participants or the weighed-in values. All dilutions and the HPLC measurements were performed at the Department of Clinical Chemistry (Karlstad, Sweden). The diluted preparations were carefully mixed to ensure even distribution of iohexol and sent to Equalis in liquid form. At Equalis the materials were pipetted into $2 \mathrm{~mL}$ tubes with $0.8 \mathrm{~mL}$ sample per tube and stored at $-20^{\circ} \mathrm{C}$ until distribution to participating laboratories. Ethical approval for preparing EQA materials from surplus unidentified pooled blood donor plasma was not required.

\section{Statistical analysis}

Interlaboratory variation, linear correlations and deviation of mean values from calculated values were calculated using Excel 2013 (Microsoft, Seattle, WA, USA). Mean values and coefficient of variation $(\mathrm{CV})$ were calculated according to the robust procedure described in the standard ISO 17043 (www.iso.org), as stated. The change in bias between the first seven EQA distributions and the seven most recent surveys was analyzed using Statistica 4.5 (StatSoft Inc, Tulsa, OK, USA).

\section{Results}

\section{CV for the different EQA materials}

The number of laboratories reporting test results varied between 27 and 34 during the study period. The majority
(77\%) of the results were analyzed using HPLC, $15 \%$ used UPLC/MS/MS and the remainder (8\%) used UPLC, HPLC/ MS/MS or "unknown methods". The interlaboratory CV for the different EQA materials varied between $2.7 \%$ and $7.1 \%$ (mean 4.7\%) for the HPLC methods, and between 1.5\% and $12.2 \%$ (mean $6.4 \%$ ) for the UPLC/MS/MS methods (Table 1 and Figure 1).

The interlaboratory CV was slightly higher in materials with iohexol levels around $20 \mathrm{mg} / \mathrm{L}$ than in higher concentrations (Figure 1). The interlaboratory CV for the UPLC/MS/MS methods was generally somewhat higher than for the HPLC methods (Figure 2).

Table 1: Interlaboratory CV for samples sent out in each external quality assessment round.

\begin{tabular}{|c|c|c|c|c|c|}
\hline \multirow[t]{2}{*}{ Material } & \multirow[t]{2}{*}{$\mathbf{n}$} & \multicolumn{2}{|c|}{ lohexol, mg/L } & \multicolumn{2}{|c|}{ Reproducibility CV } \\
\hline & & Mean & $\begin{array}{r}\text { Robust } \\
\text { mean }\end{array}$ & CV, \% & $\begin{array}{r}\text { Robust } \\
\text { CV, \% }\end{array}$ \\
\hline 2015:01/A & 30 & 116.2 & 116.3 & 4.59 & 4.65 \\
\hline 2015:01/D & 27 & 63.0 & 63.0 & 4.00 & 3.73 \\
\hline 2015:02/D & 29 & 64.2 & 64.2 & 4.29 & 4.85 \\
\hline 2015:02/B & 28 & 22.9 & 22.8 & 6.55 & 6.65 \\
\hline 2015:03/E & 29 & 81.1 & 81.0 & 4.14 & 4.32 \\
\hline $2015: 03 / C$ & 29 & 43.2 & 43.1 & 4.37 & 4.55 \\
\hline 2015:04/F & 32 & 102.1 & 101.7 & 5.81 & 5.72 \\
\hline 2015:04/D & 32 & 64.0 & 63.9 & 5.85 & 4.76 \\
\hline $2016: 01 / C$ & 32 & 43.7 & 43.8 & 5.35 & 5.93 \\
\hline 2016:01/B & 32 & 22.3 & 22.3 & 6.54 & 7.09 \\
\hline $2016: 02 / F$ & 29 & 100.9 & 100.9 & 4.12 & 4.43 \\
\hline $2016: 02 / C$ & 29 & 43.7 & 43.8 & 4.49 & 4.53 \\
\hline $2016: 03 / E^{a}$ & 29 & 82.5 & 82.3 & 3.61 & 3.62 \\
\hline $2016: 03 / C$ & 30 & 44.1 & 44.0 & 3.82 & 3.64 \\
\hline 2016:04/C & 33 & 44.8 & 44.7 & 4.68 & 3.57 \\
\hline 2016:04/B & 33 & 22.8 & 22.8 & 6.12 & 5.64 \\
\hline 2017:01/F & 31 & 103.7 & 103.1 & 6.76 & 6.08 \\
\hline 2017:01/E & 32 & 83.8 & 83.4 & 7.03 & 6.11 \\
\hline 2017:02/I & 30 & 55.6 & 55.3 & 5.24 & 4.52 \\
\hline $2017: 02 / G$ & 31 & 20.1 & 20.0 & 6.10 & 5.34 \\
\hline 2017:03/K & 30 & 99.6 & 99.5 & 5.04 & 4.81 \\
\hline 2017:03/H & 30 & 38.2 & 38.2 & 5.71 & 5.13 \\
\hline 2017:04/J & 30 & 85.4 & 85.3 & 3.84 & 4.18 \\
\hline $2017: 04 / G^{a}$ & 30 & 20.2 & 20.2 & 6.89 & 6.80 \\
\hline 2018:01/I & 34 & 54.1 & 54.4 & 5.00 & 3.34 \\
\hline 2018:01/H & 33 & 37.7 & 38.0 & 6.37 & 3.95 \\
\hline 2018:02/J & 33 & 84.9 & 84.6 & 4.64 & 3.83 \\
\hline 2018:02/H & 33 & 38.1 & 38.1 & 3.72 & 4.12 \\
\hline
\end{tabular}

${ }^{\mathrm{a}}$ One extreme value excluded. In total 10 different materials (A-J) have been used during the period. Each round consisted of two samples. Mean values and CV calculated without exclusion of possible outliers and mean and CV calculated by robust methods are presented for each sample. 


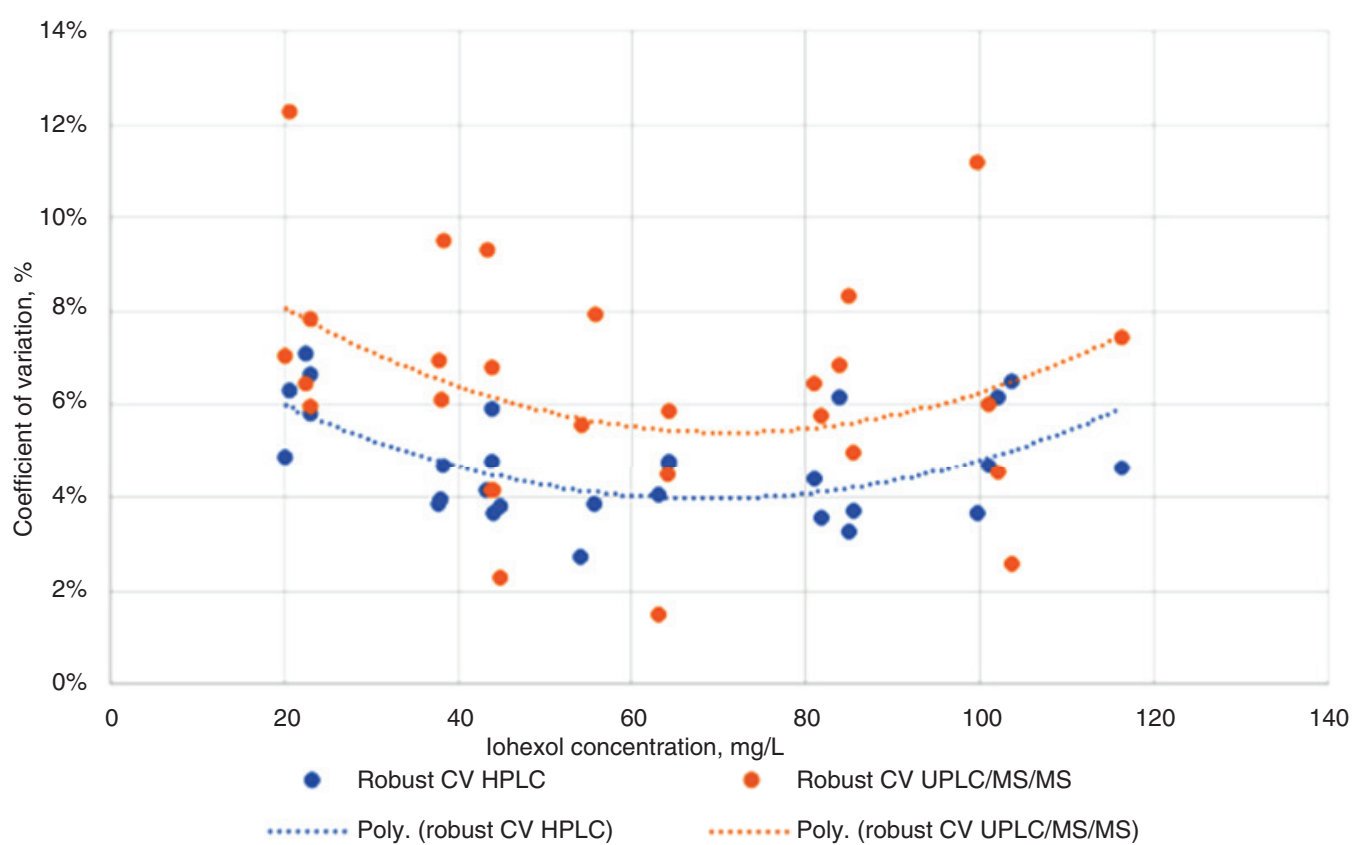

Figure 1: Mean interlaboratory CV (y-axis) vs. measured concentration of iohexol (x-axis) for laboratories using either HPLC or UPLC/MS/MS.

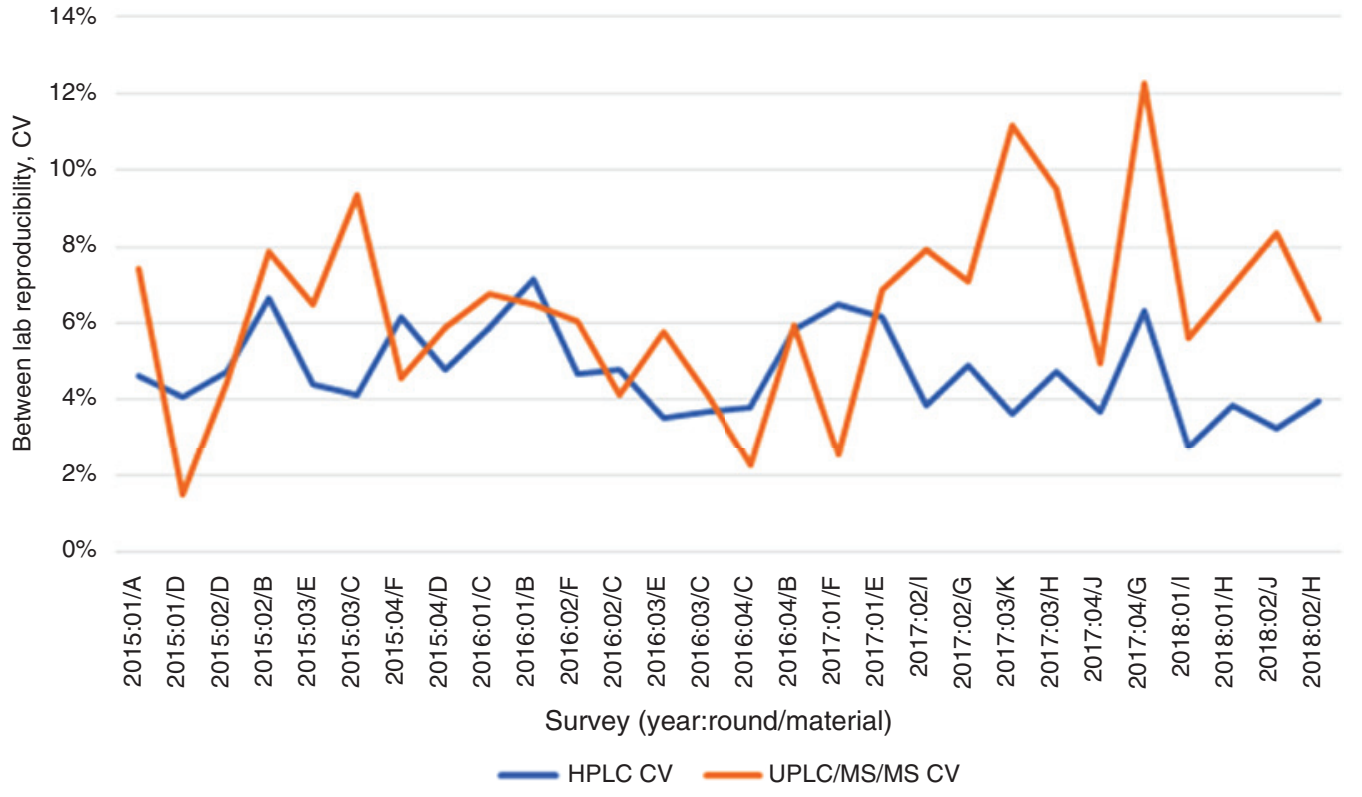

Figure 2: Between laboratory reproducibility for HPLC methods and UPLC/MS/MS methods vs. time of distribution. Since 2017, the reproducibility for HPLC methods has been better than for UPLC methods.

\section{Within- and between-laboratory variation}

Material $\mathrm{C}$ was used in five surveys and material $\mathrm{H}$ in three surveys. The data from these materials were used to compare within laboratory with between laboratory CV. The results showed that within laboratory CVs were larger than between laboratory variation (Table 2).

\section{Bias between calculated and measured iohexol values}

For materials B-F, distributed during the period 2015-2016, the mean bias was $-1.3 \mathrm{mg} / \mathrm{L}$ ( $95 \%$ confidence interval \pm 0.3 ), as compared to $0.1 \mathrm{mg} / \mathrm{L}( \pm 0.3)$ for materials G-J distributed during the period 2017-2018 ( $\mathrm{p}<0.001)$ (Figure 3). 
Table 2: Eleven different materials have been used during the study period and distributed in 14 surveys.

\begin{tabular}{lrrrrrr}
\hline Material & $\begin{array}{r}\text { Weighed } \\
\text { value, } \mathbf{m g} / \mathrm{L}\end{array}$ & $\begin{array}{r}\text { Number of } \\
\text { surveys }\end{array}$ & $\begin{array}{r}\text { Total number } \\
\text { of results }\end{array}$ & $\begin{array}{r}\text { Measured mean } \\
\text { value, } \mathbf{m g} / \mathbf{L}\end{array}$ & $\begin{array}{r}\text { Within- } \\
\text { lab CV }\end{array}$ & $\begin{array}{r}\text { Between- } \\
\text { lab CV }\end{array}$ \\
\hline $\mathrm{C}$ & 44.86 & 5 & 181 & 43.94 & 3.87 & 2.69 \\
$\mathrm{H}$ & 37.79 & 3 & 122 & 37.98 & 4.53 & 2.87 \\
\hline
\end{tabular}

Material C (five surveys) and Material H (three surveys) were used to calculate within laboratory and between laboratory CV.

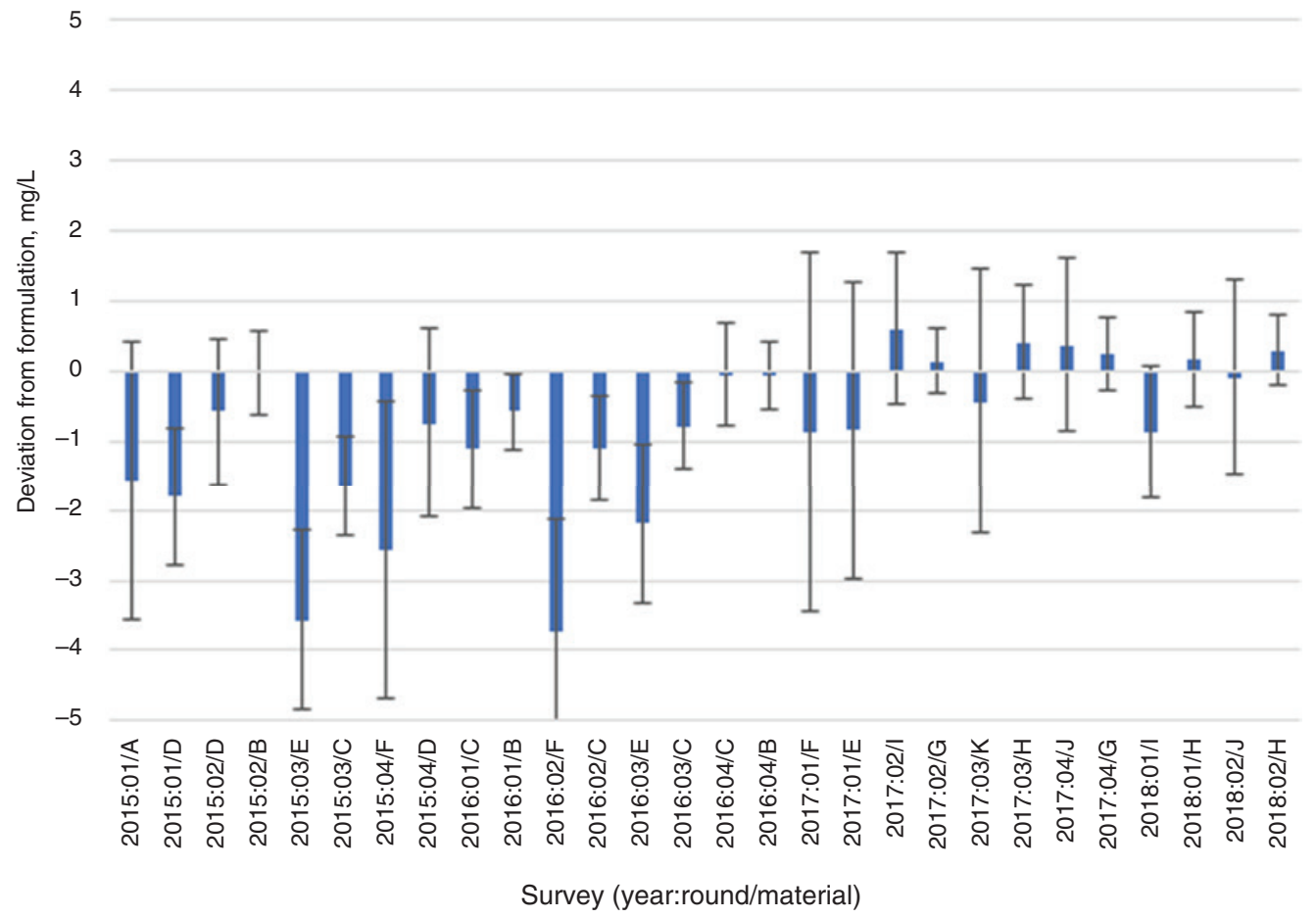

Figure 3: Bias between mean of measured iohexol concentrations and the iohexol value calculated when preparing the EQA materials. Error bars indicate $95 \%$ confidence intervals.

\section{Discussion}

GFR estimates have been made increasingly available over the last few decades. The interest can in part be traced back to the K/DOQI initiative [10] by the National Kidney Foundation, where laboratories were encouraged to provide a calculated GFR whenever a plasma creatinine was ordered; an initiative that spawned a plethora of GFR estimation formulas. An estimated GFR based upon plasma marker level in addition to a few anthropometric variables is often sufficient for daily clinical decision-making, but when the need for accuracy and precision is increased (kidney donation, treatment with nephrotoxic drugs, etc.) a measured GFR calculated from the plasma disappearance curve of an exogenous marker, e.g. iohexol, may be called for [4]. Iohexol has been used for this purpose for more than 30 years and has gained wide-spread recognition as a true gold standard for GFR measurement. Iohexol can be analyzed in plasma after administration of a small dose, and as it is non-radioactive, stable in plasma and comparatively easily quantitated using HPLC or mass spectrometry, reliable monitoring of iohexol elimination is possible. However, continuous provision of high quality GFR measurements requires the analytical process to be strictly controlled. Participation in EQA schemes ensures that both bias and precision are checked on a regular basis, and participation is also a prerequisite for the analysis to be accredited according to ISO15189. Equalis' iohexol EQA scheme is to the best of our knowledge the only available EQA scheme for exogenous GFR markers. The results in this study represent laboratories performing measurements of iohexol on a regular basis, mainly in Europe, 55\% from Sweden and $40 \%$ from the rest of Europe. The remaining 5\% are participants from Africa, Asia and the US. 
To the best of our knowledge almost all participants use Omnipaque ${ }^{\circledR}$ as standard for the iohexol measurements. Occasionally, alternative modes of calibration have been reported, with acceptable results if the ratio between the two stereoisomers has been considered and is identical with that for the substance provided to the patient. Considering the lack of certified reference materials for iohexol, the preparation of each laboratory's own calibrators and the existence of several suppliers of iohexol, the mean interlaboratory $\mathrm{CV}$ found in this study can be considered low. The mean bias between calculated and measured iohexol values was also low, demonstrating a lack of calibration issues. In addition, bias was considerably lower in the latter seven rounds, suggesting that the participants have acted on their EQA results to review their assay method to further improve performance.

In principle, different sources of iohexol standards contribute to the interlaboratory bias. This will be further investigated in future studies.

The average levels of iohexol measured with HPLC and UPLC/MS/MS did not differ, while the interlaboratory CV was somewhat higher for the UPLC/MS/MS methods than for the HPLC methods (Figure 2). Thus, the discrepancy in measurement level between HPLC and LC-MS/MS methods noted by Delanaye and coworkers [11] is not confirmed by the data in this EQA scheme.

Iohexol determinations are exclusively used for measuring glomerular filtration rate; thus, the demands for analytical accuracy will be defined by the intraindividual variation in GFR as such. As GFR cannot be studied directly, arriving at a figure entails subtracting the analytical variation from the total variation as determined by repeated measurements of GFR in the same subject. By doing so, Krutzen and coworkers found a total variation of $11 \%$, which given the analytical variation of $5 \%$ suggested an intra-individual variation in GFR of approximately 10\% [12]. The subjects were healthy volunteers with GFR in the normal range. Other workers $[5,13,14]$ have found similar or lower figures. However, none of the studies have fulfilled the Biological Variation Data Critical Appraisal Checklist (BIVAC) [15]. Despite the limitations of data, biological variation in GFR appears to be at least as high as the methodological imprecision for iohexol, which suggests that the latter does not contribute significantly to the total error of GFR measurement.

In conclusion, the EQA results demonstrate that iohexol can be measured reliably by the participating laboratories and further support the use of iohexol as a reliable GFR marker.
Author contributions: All the authors have accepted responsibility for the entire content of this submitted manuscript and approved submission.

Research funding: The Uppsala University Hospital Research Fund, Sweden supported this study.

Employment or leadership: None declared.

Honorarium: None declared.

Competing interests: The funding organization(s) played no role in the study design; in the collection, analysis, and interpretation of data; in the writing of the report; or in the decision to submit the report for publication.

\section{References}

1. Filler G, Yasin A, Medeiros M. Methods of assessing renal function. Pediatr Nephrol 2014;29:183-92.

2. Soveri I, Berg UB, Bjork J, Elinder CG, Grubb A, Mejare I, et al. Measuring GFR: a systematic review. Am J Kidney Dis 2014;64:411-24.

3. Frennby B, Sterner G. Contrast media as markers of GFR. Eur Radiol 2002;12:475-84.

4. Delanaye P, Melsom T, Ebert N, Back SE, Mariat C, Cavalier E, et al. Iohexol plasma clearance for measuring glomerular filtration rate in clinical practice and research: a review. Part 2: why to measure glomerular filtration rate with iohexol? Clin Kidney J 2016;9:700-4.

5. Delanaye P, Ebert N, Melsom T, Gaspari F, Mariat C, Cavalier E, et al. lohexol plasma clearance for measuring glomerular filtration rate in clinical practice and research: a review. Part 1: how to measure glomerular filtration rate with iohexol? Clin Kidney J 2016;9:682-99.

6. Erley CM, Bader BD, Berger ED, Tuncel N, Winkler S, Tepe G, et al. Gadolinium-based contrast media compared with iodinated media for digital subtraction angiography in azotaemic patients. Nephrol Dial Transplant 2004;19:2526-31.

7. Ye T, Xu B, Wang Z, Zhang TY, Hu CY, Lin L, et al. Comparison of iodinated trihalomethanes formation during aqueous chlor(am) ination of different iodinated X-ray contrast media compounds in the presence of natural organic matter. Water Res 2014;66:390-8.

8. Crosier AE, Henghali JN, Howard J, Pukazhenthi BS, Terrell KA, Marker LL, et al. Improved quality of cryopreserved cheetah (Acinonyx jubatus) spermatozoa after centrifugation through Accudenz. J Androl 2009;30:298-308.

9. Gaspari F, Thakar S, Carrara F, Perna A, Trillini M, Aparicio MC, et al. Safety of Iohexol administration to measure glomerular filtration rate in different patient populations: a 25-year experience. Nephron 2018;140:1-8.

10. National Kidney Foundation. K/DOQI clinical practice guidelines for chronic kidney disease: evaluation, classification, and stratification. Am J Kidney Dis 2002;39:S1-266.

11. Delanaye P, Jouret F, Le GC, Cavalier E. Concordance between iothalamate and iohexol plasma clearance. Am J Kidney Dis 2016;68:329-30.

12. Krutzen E, Back SE, Nilsson-Ehle I, Nilsson-Ehle P. Plasma clearance of a new contrast agent, iohexol: a method for the assessment of glomerular filtration rate. J Lab Clin Med 1984;104:955-61. 
13. Perrone RD, Steinman TI, Beck GJ, Skibinski Cl, Royal HD, Lawlor $M$, et al. Utility of radioisotopic filtration markers in chronic renal insufficiency: simultaneous comparison of 1251-iothalamate, $169 \mathrm{Yb}$-DTPA, $99 \mathrm{mTc}$-DTPA, and inulin. The modification of diet in renal disease study. Am J Kidney Dis 1990;16:224-35.

14. Levey AS, Greene T, Schluchter MD, Cleary PA, Teschan PE, Lorenz RA, et al. Glomerular filtration rate measurements in clinical trials. Modification of Diet in Renal Disease Study Group and the Diabetes Control and Complications Trial Research Group. J Am Soc Nephrol 1993;4:1159-71.

15. Aarsand AK, Roraas T, Fernandez-Calle P, Ricos C, Diaz-Garzon J, Jonker $\mathrm{N}$, et al. The biological variation data critical appraisal checklist: a standard for evaluating studies on biological variation. Clin Chem 2018;64:501-14. 